\title{
Investigating Touchscreen Accessibility for People with Visual Impairments
}

\author{
David McGookin, Stephen Brewster, WeiWei Jiang \\ Department of Computing Science \\ University of Glasgow \\ Glasgow, G12 8QQ \\ \{mcgookdk, stephen\}@dcs.gla.ac.uk \\ www.dcs.gla.ac.uk/ mcgookdk
}

\begin{abstract}
Touchscreen computing devices such as the iPhone are becoming more common. However this technology is largely inaccessible to people with visual impairments. We present the results of a requirements capture study that illustrates the problems with touchscreen accessibility, and the choices visually impaired people make when choosing assistive technology. We investigate ways of overcoming touchscreen accessibility problems by comparing a raised paper overlay touchscreen based MP3 player, with a touchscreen gesture based player. Twelve blindfolded participants, and one visually impaired person, were able to operate both players, though there were problems with short impact related operations in the gesture player. From our results we provide guidelines for future designers, to help them exploit the potential of touchscreen technology for visually impaired people.
\end{abstract}

\section{Categories and Subject Descriptors}

H5.2 [Information Interfaces and Presentation]: User Interfaces - Auditory (non-speech) feedback, Evaluation/methodology, Input devices and strategies (e.g., mouse, touchscreen), Interaction styles (e.g., commands, menus, forms, direct manipulation), User-centered design.

\section{General Terms}

Design, Economics, Experimentation, Human Factors, Standardization.

\section{Keywords}

Visual Impairment, Blind, Touchscreen, Non-Speech Sound, Speech, Accessibility, Mobile Devices, Universal Design

\section{INTRODUCTION}

An increasing amount of mobile consumer technology is beginning to incorporate a touch sensitive screen. Devices such

Permission to make digital or hard copies of all or part of this work for personal or classroom use is granted without fee provided that copies are not made or distributed for profit or commercial advantage and that copies bear this notice and the full citation on the first page. To copy otherwise, or republish, to post on servers or to redistribute to lists, requires prior specific permission and/or a fee.

NordiCHI 2008: Using Bridges, 18-22 October, Lund, Sweden

Copyright 2008 ACM ISBN 978-1-59593-704-9. \$5.00 as Apple Inc's iPhone (see Figure 1) and iPod touch, have gained widespread consumer acceptance, and allowed easy interaction with media on mobile devices, without the need to allocate valuable space to dedicated control surfaces. Other manufacturers are now also producing devices which rely on touchscreens as the sole input/output mechanism [1] [2]. In addition, an increasing number of public access terminals, such as bank ATMs (automated teller machines), ticket machines and information points, are moving away from incorporating physical control panels and buttons and towards touchscreen technology.

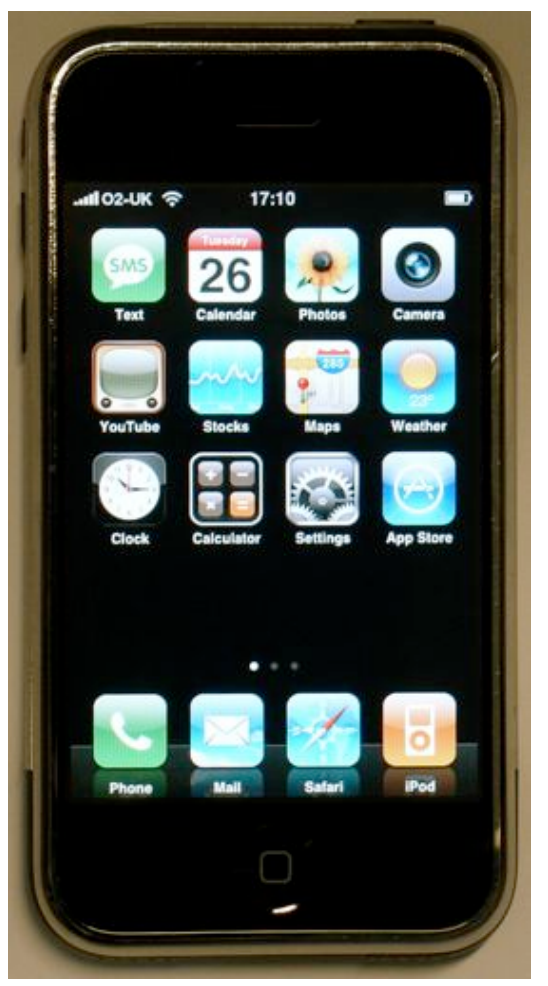

Figure 1: Consumer electronics such as Apple Inc's iPhone are increasingly employing touchscreen technology.

Unfortunately, the increasing use of touchscreen technology presents significant problems to the European Union's 2.7 million blind and 27 million visually impaired citizens [3]. Unlike the controls on a standard mobile telephone or public access terminal, that can be easily felt through touch, touchscreen technologies do not provide any tactile distinguishment between controls and 
display space. Whilst a visually impaired person can learn the locations and functions of tactile control panels on current mobile telephones and public access terminals, attempting to do the same with touchscreen based devices is much harder, due to the lack of tactile distinguishment between virtual buttons and surrounding surfaces. This affects the degree of independence that a visually impaired person can enjoy when trying to use a touchscreen based device. This lack of accessibility may also contravene recent legislative programs such as the American Disability Act [4], that require devices to be accessible to people with visual impairments. We argue in this paper that although visually impaired people find touchscreens to be inaccessible, the increasing use of touchscreen technology presents a significant opportunity to improve several aspects of assistive technology, allowing good accessibility to be incorporated into the same devices, and on an equal footing, to interaction for sighted users. We argue this through the results of a questionnaire based study on the use of everyday technology by visually impaired people, before carrying out a motivated study comparing possible interaction techniques on two touchscreen based MP3 players.

\section{ISSUES WITH TOUCH SCREEN ACCESIBILITY}

To identify more fully the problems of touchscreen accessibility, we carried out an email based questionnaire. We chose to disseminate by email as it is difficult to gain access to a large group of appropriate respondents by traditional means. As not all participants would be able to read Braille, paper based dissemination would need to be augmented by audio cassette, $C D$ etc. This would raise issues in how participants would provide their responses. Therefore email was the most reliable option.

We disseminated electronic copies of the questionnaire to visually impaired contacts, visually impaired mailing lists, and local associations for visually impaired people. We have also been interviewed on a local radio station for visually impaired people (www.insightradio-net.com), who have helped distribute the questionnaire. We employed snowball sampling [5], where participants are asked to forward the questionnaire to others whom they know and would be willing to complete it. Due to the distribution medium, the responses are from technologically competent users, and therefore may not be typical of others with visual impairments. However, the responses do identify those users who are willing to engage with technology, and the results represent one of the end points of user experience. We left many of the questions open ended. We asked users for basic demographic information, including details of their visual impairment. This was followed by sections asking about leisure time, and the activities participants engaged in, followed by the technologies that they used in these activities. For each of these technologies we asked participants to rate the appealing and frustrating features of each device, and to discuss the features that they would find appealing if incorporated into these technologies. As the questions were open-ended (e.g. "What accessibility aids do you use to access computers?"), some of the responses were incomplete or missing details. We overcame this by arranging follow up telephone interviews, or by more detailed follow up questionnaires, distributed by email, tailored to the responses of each user. We received seventeen questionnaire responses. These were analysed, and the findings compiled into a "mind map", to reveal a picture of technologies and their use.
Three participants rated themselves as visually impaired and having some residual vision rather than blind. Seven participants had been blind since birth. Four participants had become blind progressively, with a significant number of years having either full sight or being visually impaired. A further three participants were blind, but declined to provide further details. Participants were asked to provide their age in one of five categories. The median age range was 36-45 and the mode age range was 46-55. Respondents had a wide range of occupations, including charity fundraising managers, university lecturers and computer programmers, as well as being retired or unemployed.

\subsection{Questionnaire Results}

All responses discussed how every day activities were supported (or not) by technology. Participants discussed a wide range of technologies and services that they used to carry out common activities, such as watching TV, listening to music, communicating with friends and reading books. Whilst in most cases each participant named different devices to accomplish the same task, there were common themes around the reasoning why respondents chose particular technologies. These are outlined in the following sections.

\subsubsection{Appropriated vs. Assistive Technology}

An interesting issue to arise through the responses, was the way in which technologies chosen were divided between specially designed assistive technologies, and appropriated "mainstream" devices. For example, choosing between a specially designed mobile telephone and a cheap, but large and simple, standard mobile telephone. We identified that this is not necessarily an "either or" decision. Many participants used both assistive and appropriated devices to complement each other, with one device overcoming the limitations of the other. In this section we will look at some of the issues that drove these decisions.

\subsubsection{Cost}

Dedicated assistive technology tends to be more expensive than appropriated technology. This is not surprising, since the numbers of units that are sold of assistive technology devices is much lower than those sold of a "mainstream" device for the same purpose. As one respondent noted: "An awful lot of assistive technology is far too expensive. Speech mobile phones are far too expensive. A normal sighted person can buy a phone for under $£ 20$ which will have the ability to send text messages whereas we have to have something added on or buy a specially made phone for over £300." Lower demand has knock-on effects, such as a lack of competition in the marketplace leading to slower revisions of products. One example is the DAISY Player [6]. DAISY is an open standard for the storage of audio books, allowing nonsequential access as might be required for text books, information leaflets etc. However, few devices to play the books are available. One that many respondents discussed was the Plextor Plextalk [7] (see Figure 2). Whilst this device provides non-linear access, such as might be required with government information leaflets or reference books, it is also expensive, retailing at $£ 225$ $£ 530$, lacks portability (weighing $1.2 \mathrm{~kg}$ ), with a battery lasting about $5 \mathrm{hrs}$. In comparison, similar mainstream MP3 players weigh a few grammes, cost around $£ 30$ and can last for almost $24 \mathrm{hrs}$ on a single charge. However, these do not provide DAISY book access. Respondents noted that whilst they liked the ability to play books they wished "the PlexTalk were more portable and used something like AA batteries". 


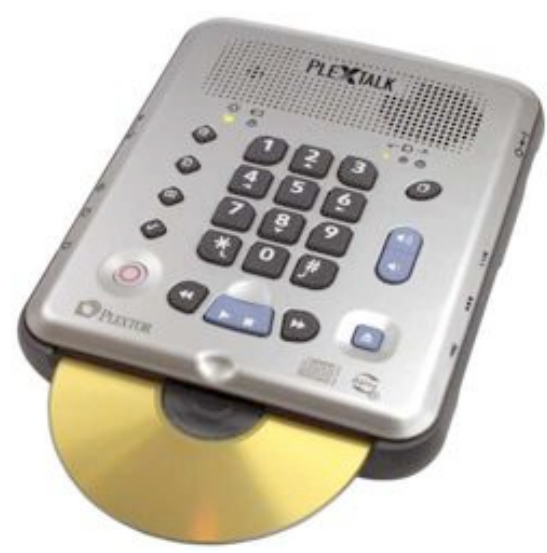

Figure 2: The Plextor Plextalk CD and DAISY book player.

\subsubsection{Feature Access}

Another issue when deciding on appropriated technology is that accessibility is not a binary state. Whilst some features of devices may be accessible to a visually impaired person, other features, which may be useful, would need to be sacrificed. For example, most modern digital media players (such as the iPod or Creative Zen), have spatially located buttons for basic features (such as play, pause and fast forward) whose location can be learned (see Figure 3). However, navigating the menus via the visual display is difficult, as the menus must be learned. Menus may also change as data is added or removed from the device requiring re-learning. As one respondent mentioned: "I see other family members using MP3 players that I would love to be able to access! They are so small and so portable, and I think they can get ten thousand songs on these players! I wish I could access all of the menus that can be gotten on these types of players and have access to the extreme portability." Many of the respondents noted that they had used more basic MP3 players, such as the Creative Zen Stone (see Figure 4) and the iPod shuffle, which were described as "wonderful, because I don't have to read anything. It is something I can do completely by touch".

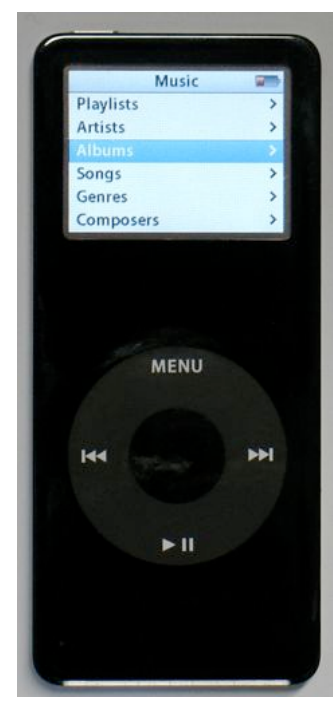

Figure 3: iPod by Apple Inc has spatial and tactile distinguishment of buttons, but screen based menus are inaccessible.
Such reasons were also mentioned as to why many respondents were still using compact cassette players and basic compact disc players: "I always choose cassette and CD players with good tactile controls which are easy to learn and remember". We identified these same issues with mobile telephones, where many respondents had mobile telephones, but these were only lightly used, with simplicity and tactile features such as large distinguishable buttons favoured over functionality: "I have a mobile phone, for emergency use only, .... I bought the cheapest and simplest phone I could find, and it has well defined buttons. I can't use all its features, but I don't want to. Many mobile phones now have the tiniest controls.".

\subsubsection{Application to Touchscreens}

A particular feature that participants discussed, was the increasing role of touchscreen interaction in both portable technology and everyday devices. Whilst we have previously mentioned devices such as the Apple iPhone, participants pointed out a range of devices and activities that are becoming more dependent on touchscreen based interaction. Examples cited included gym equipment, cash machines and ticket machines. Users make informed decisions as to using specifically made assistive technology, which provides easy access to all device functionality, but is more expensive, and mainstream appropriated technology that is lower cost but has poorer accessibility, and some features that cannot be used. However, as many more of our everyday technologies such as phones move to incorporate touchscreens, visually impaired people may no longer be able to access any of the functionality, and loose this valued choice.

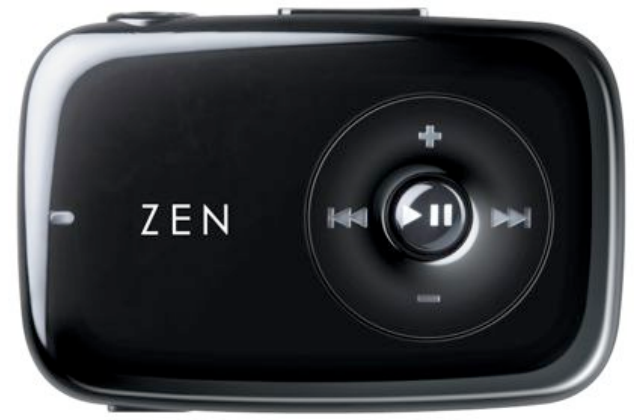

Figure 4: The Creative Zen Stone MP3 music player.

This is more of a concern when the devices are those which a person has no choice but to access, such as ticket machines or bank ATMs. Organisations are increasingly using touch based technology to reduce maintenance costs. However such organisations may also have legal requirements to make their touchscreen devices accessible. As there are no physical tactile controls, it is unclear how this can be achieved. We believe that ultimately greater accessibility can be provided through touchscreen based devices than is currently possible through existing button based interfaces. In the following section we will expand on this argument, before describing an experimental study comparing and contrasting two approaches to non-visual touchscreen accessibility. 


\section{UNIVERSAL TOUCHSCREEN ACCESS}

When constructing a conventional electronic device such as a mobile phone, the position of physical controls is fixed and cannot be changed after production. Any applications that run on the device must be adapted to fit with the physical controls available. However, on a touchscreen device the position of buttons and controls is dynamic, and these can be changed based on the application the device is running. A telephone keypad can be used when making calls, with a more appropriate interface created for video or audio playback. In the discussion of appropriated vs. assistive technology, participants discussed that many current mobile phones had very small controls, or that they liked the DAISY music player due to the large controls. If the advantages of touchscreen devices could be used here, an alternate large button based interface for people with visual impairments could be created as a software preference, whilst having a more "mainstream" interface with the contact pictures and visual interaction that a sighted user would wish. Allowing this would reduce the need to produce low numbers of expensive, but desirable devices, such as the DAISY player. In other words, the difference between dedicated assistive technologies and appropriated mainstream technologies can be reduced, with dedicated interfaces produced to suit both mainstream sighted users, and those with visual (or indeed other) impairments.

The problem with this approach of course, is how a visually impaired person can interpret the controls. The buttons and other controls on a touchscreen cannot be felt in the same way as a real physical interface. This leads to many of the problems of touchscreen interaction for people with visual impairments. In the remainder of this paper we explore how better interaction with the touchscreen can be achieved, and compare two methods to provide this. In doing this we have focused on techniques that can be accommodated through modified software, with no expensive physical or permanent modification required to the device. In cases where access is improved to public terminals, those terminals would need to be used by both visually impaired and sighted users, precluding such modification.

\section{TOUCH SCREEN MP3 PLAYER}

In order to investigate how touchscreen interaction could be adapted to be more accessible to users who are blind and visually impaired, we decided to compare two different techniques that could be practically applied to current touchscreen devices. From the questionnaire based survey, listening and playing music is a popular pastime, so the techniques chosen were applied to a touchscreen MP3 player. With devices such as the Apple iPhone, iPod touch, as well as touchscreen smart phones becoming more popular and prevalent, such an application for investigation is both practical and relevant. As mentioned, two techniques were chosen that rely on current touchscreen technology, and which could be practically implemented without physical hardware modification to the device. Both of the techniques have been used before, but not to investigate accessibility of touchscreen devices. In the following subsections we outline the techniques chosen, as well as how these were implemented to create two MP3 players that ran on a Dell Axim x51v PDA platform.

\subsection{Overlaid Buttons Player}

The first technique applied to the MP3 player, was to overlay the visual touchscreen display with a raised paper control panel which incorporated tactile buttons that overlaid the virtual buttons on the touchscreen (see Figure 5). Raised paper diagrams are a common way in which people with visual impairments can access information through touch. For example, they are commonly used in schools to teach about graphs [8]. It is also a technique that has been successfully incorporated in prior information displays for people with visual impairments. Challis and Edwards [9] constructed a musical annotation system for a visually impaired person incorporating a commercial tablet touchpad with a custom plastic tactile overlay. The tactile overlay represented constant elements of the interface, such as musical measures, and used audio feedback to present more dynamic and changing information. They identified several guidelines for designing overlays, such as avoiding large empty areas, that we have used in our player. Wall and Brewster [10], extended this idea using physical guides (compact discs) overlaid on a standard graphics tablet, to allow visually impaired users to browse pie charts. The user moved a pen around the outside of the $\mathrm{CD}$, and received information through both speech and non-speech audio about the current segment of the pie chart. The user could also receive tactile information through a pin array in his or her non-dominant hand. Wall and Brewster proposed that by using RFID (Radio Frequency Identification) tags, different overlays could be used dependant on the way the underlying data should be viewed. For example, different overlays could provide a static representation of the axis of a bar chart or line graph, and the system could present the data in an appropriate way for that representation, based on the attached RFID tag.

In addition to research using this technique, commercial systems, such as the T3 talking tablet learning aid [11], are becoming available. The T3 allows tactile diagrams to be augmented with audio feedback. A tactile diagram is positioned over a touch sensitive graphics tablet, and produces speech and non-speech audio feedback to support users' exploration. For example, a tactile map of the world may produce the names of countries or continents on request. To indicate to the computer which overlay is used, a sequence of small bars in the corner of each diagram must be pressed by the user before interaction.

All of these techniques share similar features, such as using a common control layout for all interactions, or using techniques to allow simple or automatic detection of the overlay used. Similar techniques could be used for public access terminals such as ticket machines, where an RFID tag, attached to the overlay, could be detected by the terminal, which could switch into a control panel "compatibility mode".

In our implementation of this technique for the MP3 player, a "control panel" the same size as the PDA touchscreen was created in a computer drawing program. On this panel, control buttons were drawn in locations corresponding to the virtual buttons on the PDA screen. By printing the control panel on special swell paper, and passing this paper through a thermal printer, the control buttons raise above the paper surface creating a tactile panel that can be easily felt. Buttons were approximately $1.5 \mathrm{~cm}$ in the longest dimension, and separated by at least $0.5 \mathrm{~cm}$ from each other. This panel was then temporally attached onto the PDA screen (see Figure 5). A user can feel the panel attached to the display. Buttons are given different shapes to be distinguishable, and by increasing pressure on the raised paper control, the virtual touchscreen button underneath can be activated. 


\subsection{Gesture Driven Player}

The second technique investigated is the use of gestural interaction. Thanks to the iPhone, gestural interaction with touchscreens is common for sighted users, allowing them to "pinch" their photographs to zoom in or out, and "flick" between one photograph and another. However, gestural interaction has also been studied using other sensing devices such as accelerometers, joysticks and touchscreens.

Pirhonen, Brewster and Holguin [12] investigated how gestural interaction could be used on a PDA MP3, player, when the user is "on the move". They identified that when using gestural interaction, users walked at a rate closer to their personal preferred walking speed than when using the in-built PDA media player application. Users also recorded lower workload on NASA TLX scales when using the gestural interface. Pirhonen et al. however did not evaluate their system on visually impaired people.

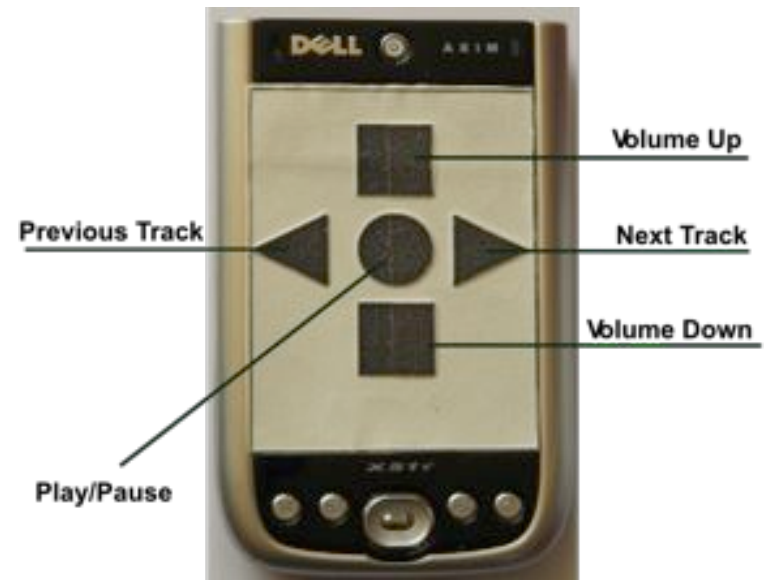

Figure 5: An image of the raised paper MP3 player showing the overlaid raised control panel.

There is an overall lack of research investigating gesture based interaction involving people with visual impairments. Klok et al. [13] have considered creating and teaching gestures to visually impaired people. Their work sought to overcome the problem of training on the gestures. By using a Sensable Technologies' PHANTOM force feedback device [14], they trained participants on 28 gestural commands. Participants were "dragged" around in space by the PHANTOM as a way of teaching the gestures. Participants then had to select seven of the gestures to represent simple operations in a user interface, such as open/close, select and delete. Participants then, on demand, had to perform gestures they had chosen for particular commands. These were recorded for analysis. Klok et al. noted that participants tended to pick similar gestures for the same operations. For example, the previous and next commands were always represented by left and right directional gestures. However, some of the command mappings were not so consistent, such as the select command, where various gestures were chosen. Care therefore should be taken in choosing gestures so that they are appropriate for the types of operations they control, otherwise lengthy training will be required.

Crossan and Brewster [15], have investigated teaching shape information using automatic playback with a PHANTOM Omni haptic device and dynamic audio feedback. In their system, two users (one sighted and one visually impaired) collaborated to draw a 2D shape. The sighted person described the shape, and the visually impaired person drew the shape using the PHANTOM. Audio feedback was provided to indicate the position of the PHANTOM device in relation to the drawing surface boundaries. In cases where the object to be drawn was complex, and difficult to describe, they found that direct manipulation of the PHANTOM by the sighted user was helpful to communicate information. They also discussed how other forms of non-speech audio could be used to delineate if the drawer was being moved by the sighted user, or experiencing other haptic cues.

Wobbrock and Myers [16] have considered the use of Unistroke crossing based gestures for people with motor impairments. They found that using unistrokes via a trackball allowed for significantly higher text entry rates in comparison to an onscreen keyboard. Leonard et al. [17] have considered how different graphical factors such as icon size, can be adapted to make a PDA more usable for those with low vision. They identified that auditory feedback was useful in improving dragging performance for those with the most serious impairments, whilst not degrading performance for those with less serious, or no impairments.

Whilst this work indicates the usefulness of gestural interfaces by visually impaired people, none directly deals with the problems identified of using a touchscreen device with no vision. Special force feedback technologies, such as by Crossan and Brewster [15] and Klok et al. [13] are used, or some residual sight is assumed, such as with Leonard et al. [17]. Many of these technologies and techniques may not be possible in the kinds of situations (such as using ticket machines) that we discuss here. Our touch based MP3 player is therefore based around that of Pirhonen et al's system, as their gestural interaction has been heavily evaluated with sighted users. We can therefore consider how well the gestures can be used without sight.

In this version of the player, the entire visual display is used as a gestural control panel. By touching the display and dragging a finger across it, functions of the player can be activated. The gestures used are simple. Dragging a finger from left to right on the screen, causes the next track in the playlist to be selected, dragging from right to left causes the previous track to be selected. Dragging from top to bottom causes the volume to be lowered, and from bottom to top the volume is increased. Tapping the screen activates the play/pause function (see Figure 6). Note that the gestures do not need to be performed on any particular part of the display, or need to extend as far as the arrows in Figure 6 indicate, just in the directions indicated.

\section{BLINDFOLDED STUDY}

In order to evaluate the relative merits of both approaches, we carried out a comparative quantative evaluation. When carrying out such experiments it is important that all of the users are derived from the same population. Carrying out evaluations with visually impaired people is difficult, as the term "visual impairment" covers a range of impairments from poor sight to full blindness [18]. Additionally, the length of visual impairment is important. Many people may be born blind (congenitally), meaning that they have no experience of the sighted world, whereas other people may have become blind through illness or accident later in life (adventitiously). These issues can vastly affect the performance of participants and the results obtained. There is no particular solution to overcome this problem, and any solution involves compromise. We have taken the approach of carrying out a quantative study with blindfolded sighted users. 
These users are most like people who have become blind later in life due to illness, or an accident that has deprived them of vision. We follow this study with a more qualitative evaluation with one blind user, where the two interfaces are compared in a more informal way to consider the validity of the blindfolded results.

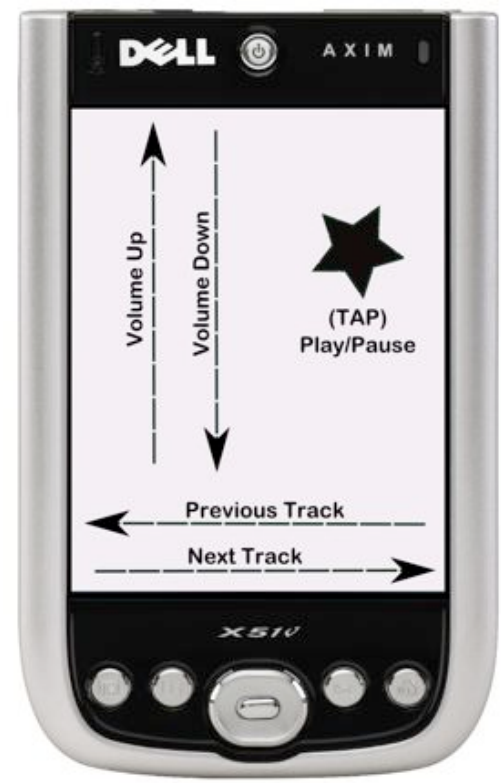

Figure 6: An illustration of the gestural MP3 player and the gestures used to control it.

\subsection{Procedure}

Twelve participants, aged between 22 and 34, were recruited to carry out the experiment. Four were female and eight were male. All participants were either undergraduate, or postgraduate students, at the University of Glasgow, and were not paid for participation. Participants completed a sequence of simple tasks using both of the MP3 players. The order in which players were used was counterbalanced to avoid learning effects. Each player allowed the user to move through, and operate over, a playlist of songs. When traversing the playlist, spoken track names were used whenever the user moved to the previous or next track. No specific audio was used to indicate play/pause or volume change. An additional sound was used in the gesture based player to indicate that a gesture had not been recognised. We assume that the implicit audio feedback, such as an increase in volume as the user increased the volume, or the audio ceasing on a pause operation, would be enough to confirm that these operations had been completed.

Before carrying out the tasks, the participant was introduced to the MP3 player used in the condition. The participant was shown the functionality and how to operate the player. This training phase was carried out without wearing a blindfold. We also kept this phase short, as in a real world scenario extensive training would not be possible for such consumer electronic devices. After the training phase, the participant donned a blindfold and was verbally instructed to carry out the tasks using the player (see Figure 7). Participants were both audio recorded, and their interactions were logged in the player software. Participants completed the tasks on both conditions before being debriefed and comments obtained.

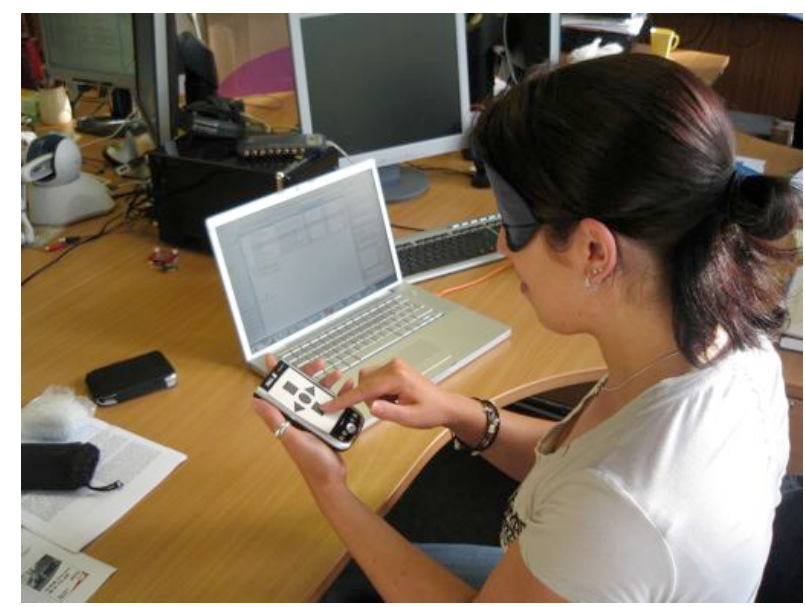

Figure 7: Participant completing the raised paper condition.

\subsection{Results}

\subsubsection{Quantative Results}

Participants were asked to complete a total of 6 tasks in order. All of which are common basic digital music player operations. Participants were asked to:

- $\quad$ find and play a specific track from the playlist.

- $\quad$ skip forward two tracks.

- $\quad$ pause and then play the current song.

- move to the previous track

- find the last track in the playlist

- $\quad$ adjust the volume

Due to an error in the logging software, accurate data for adjusting the volume cannot be reported. Data for the mean time taken for completing each of the other tasks in both conditions is shown in Figure 8 . The data shown in Figure 8 were analysed using paired t-tests. For song selection $(\mathrm{p}<0.001)$, moving forward tracks ( $\mathrm{p}<$ $0.121)$, play/pause $(p<0.045)$, go to previous track $(p<0.040$ and go to last track $(\mathrm{p}<0.030)$, performance was significantly faster in the button overlay condition.

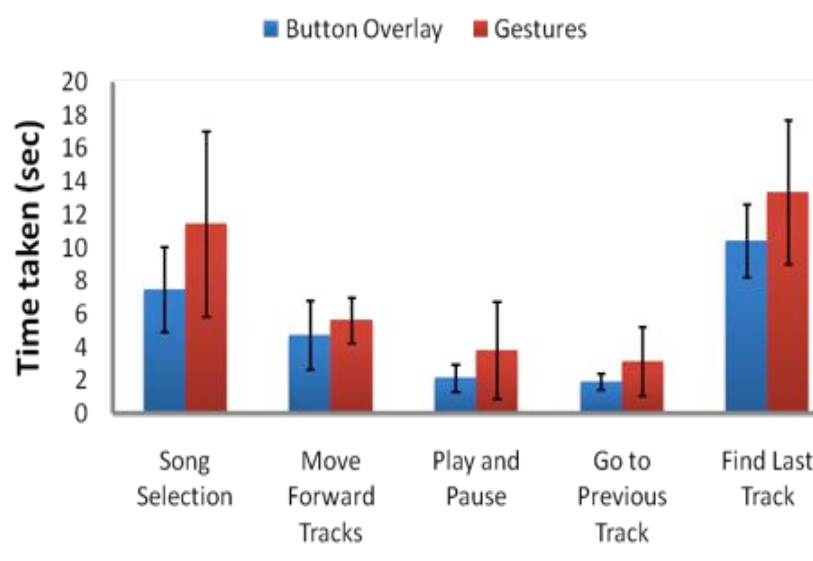

Task

Figure 8: Graph showing mean times taken for each of the tasks (excluding volume adjustment) for both conditions. Shown with standard deviations. 
As well as time taken, we also recorded the errors made by users when carrying out the tasks. We classify an error as a user operating an incorrect or unnecessary function of the player whilst carrying out a task. For the buttons condition, this was the number of tasks an incorrect button was pressed, and for the gesture condition, the number of tasks where an incorrect gesture was performed. Across all participants and trials, there were 3 occasions where an incorrect operation was performed on the buttons condition, versus 18 occasions on the gesture condition, out of a total of 72 tasks. A breakdown of errors for the gesture condition is shown in Figure 9.

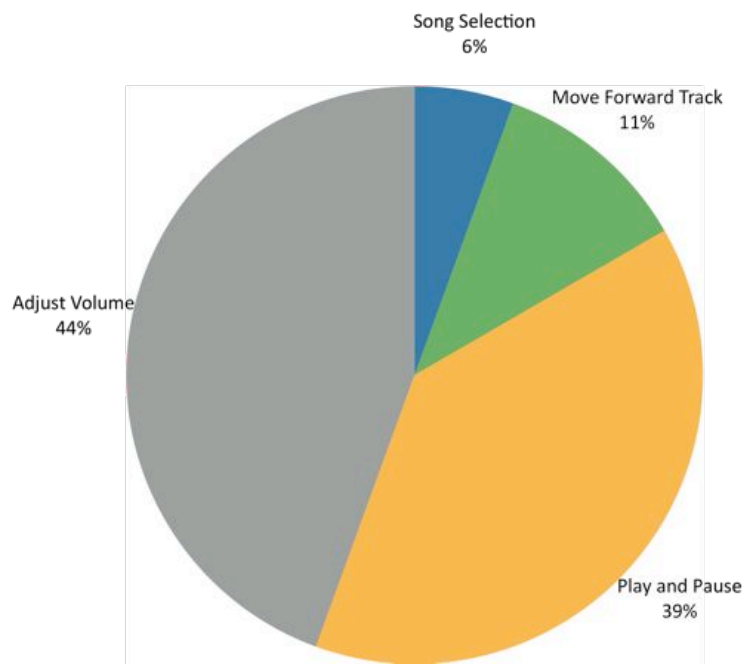

Figure 9: Breakdown of errors by type for the gesture condition.

\subsubsection{User Comments}

In addition to quantative data being collected on errors and time taken to complete the tasks, we also interviewed participants on their thoughts and experiences of both interfaces. Due to a recorder malfunction we only recorded the comments of 9 of the 12 participants. All of these nine participants expressed a preference for the buttons interface over the gesture based interface. Reasons explaining this, and the error results in the gesture condition, were obtained from these comments.

\subsubsection{Problems with Gestures}

There were several problems with the use of gestures by the blindfolded participants. Many complained that gestures were "misinterpreted" by the system. Notably, participants complained that a play/pause gesture had been completed when another gesture, usually the volume modification, had been intended, with one saying: "play and pause is easy to confuse with the volume gesture". Another participant noted: "the problem is that my command cannot be recognized correctly so that when I want to set the volume, it always recognizes that command like I want to pause". These comments are supported by the error results of Section 5.2.1, where $39 \%$ of errors involved the user attempting the play/pause gesture, and $44 \%$ of errors involved the user attempting the volume gesture. There are several reasons why this could have occurred. Notably the lack of visual feedback over how far the user was above the touchscreen, could have caused the user to interact with the screen at unexpected times, making it easier to slip over the surface causing a volume gesture rather than a play/pause gesture. Alternately, it would be easy to accidently touch and cause a play/pause gesture. Pirhonen et al. [12] did not evaluate their player on visually impaired users, but did have users wear the device on the hip whilst walking, which would have made it difficult to see the screen. They noted a similar issue, with accidental taps on the screen activating play/pause gestures.

Another factor that was problematic with the gestures, was the time taken to become comfortable with them was felt to be greater than with the button interface. Whilst some of this could be down to the novelty of the gestures, "I think it started off difficult but you get to grips with it quite quickly", some problems could be due to the reliance on memory to recall the gestures rather than the information being tacit (in the world) such as with the buttons interface: "you can understand the buttons by their directions".

Overall however, participants found the gestures usable apart from the issues of volume and play/pause functions: "It's not hard when you get used to it and seems to work quite well".

\subsubsection{Issues with the Buttons}

As participants overall preferred the buttons condition, there are fewer issues to report. The buttons having different shapes was useful, but a few of the participants felt that the distance between the buttons was too small, and that they should be moved to be further apart. Buttons were approximately $1.5 \mathrm{~cm}$ in the longest dimension, and separated by at least $0.5 \mathrm{~cm}$. One participant noted that the separating space between buttons made it difficult to distinguish them from each other. This practically means that it may not be possible to adapt an existing application simply by creating a tactile screen overlay for the existing visual interface, as the buttons and controls on the touchscreen will be too small to be easily adapted.

Another point that was noted, was the lack of "travel" on the buttons as they were pressed. One participant noted that he would "like more indent on the buttons so you feel as if they are being pressed. Sometimes the button presses are recorded too fast." We used a simple raised paper overlay, which due to the way the paper raises when heat is applied, makes the controls slightly compliant. However, it is interesting that participants may have expected the controls to operate as "real world" buttons, in spite of their awareness of using a touchscreen overlay on the device.

\section{VISUALLY IMPAIRED EVALUATION}

The evaluation involving sighted blindfolded participants yielded useful information about both touchscreen accessibility techniques. However to confirm and further consider the issues raised, we carried out a more informal qualitative evaluation with one blind individual. Our participant was a middle aged man who has been adventitiously blind for several years. He works as a transcription officer for the Royal National Institute for the Blind in the UK, and has experience in the issues surrounding DAISY audio books previously discussed. He was also one of the questionnaire respondents discussed in Section 2.

The evaluation was carried out informally, with the participant being shown each of the players in turn, and allowed to use it whilst providing his thoughts and views. As with the blindfolded participants, the buttons player was preferred. On using the gesture based player the participant had several instances when trying to perform a play/pause gesture (tap) actually performed a volume up or down gesture due to his finger skidding on contact with the surface of the touchpad. This mirrors the results discussed in Section 5.2.2.1. In order to try to overcome this, the 
participant tried using the PDA stylus to perform the gestures. Whilst gesture performance improved over time, performing the play/pause gesture was very unreliable. In addition, the participant found it difficult to be aware of his relative location on the screen, e.g. how far from the top or bottom boundary of the PDA screen he was: "What I find difficult is that it is hard to relate where you are, up, down, left, right. In my head I don't know where the stylus is to the screen, or when you are getting closer and closer." This was not a particular problem in our system, as gestures were not dependant on the location they were performed, and only required consistent movement in a single direction. However, the functionality of our player is limited. Adding functionality will require more gestures, and lack of awareness of location on the screen may become more of an issue. For example, drawing arrows on the screen (one of the gestures proposed by Klok et al. [13]) requires such awareness to be performed properly. Our participant also identified the issues discussed by both Crossan and Brewster, [15] and Klok et al. [13], on how users would be trained on the gestures, especially if the device was consumer electronics, such as an MP3 player, which precludes individual training, and the user had to operate the device "out of the box".

As already stated, the buttons player was preferred. The shape and size of buttons were not directly commented upon, so in line with the blindfolded comments, we believe that these are at least appropriate - the visually impaired user did not raise issues over button spacing that a few of the blindfolded participants raised. However, several issues surrounding the design of the control panel used were raised. The participant wanted a "reference" point to establish his location in the control panel. He noted that this was a problem with several other types of technology, such as home security systems, where buttons tend to be small and located under sections of overlaid plastic, so there is no tactile distinguishment between the buttons and the rest of the panel. $\mathrm{He}$ noted that is such cases he used "bump ons" - small adhesive plastic bumps that could be stuck to controls. We attached a "bump on" to the central play/pause button on the control panel (see Figure 10), and the participant subsequently found it easier to determine his location on the panel and to return to a "home" location for further interaction.

The participant also proposed several other modifications that may allow users to gain a quick overview of the control panel. As discussed by Kildal [19], gaining an overview of a dataset, or as in this case a control surface, is amongst the first things that a visually impaired person needs to do. It is important that during this phase, controls are not accidentally activated, which could occur in the buttons player. To overcome these issues the participant proposed that the control panel be inverted. That is, instead of the button controls being raised, the surrounding surface is raised, so that the controls are recessed in easily discriminable shaped holes. He continued to note that the edges of these holes could be further raised to make the shapes of the holes more easily discernable. It is difficult, with the raised paper technology available to us to do this. However, Challis and Edwards [9] have recommended that using different levels of relief is effective, and can be achieved by using vacuum formed plastic.

In addition to the comments on each of the players, our participant discussed more general issues relating to both of the players. The comments primarily addressed the issue of what feedback should be provided for user commands, and when this should be provided. In both players speech feedback is provided only when navigating a playlist, so that as the user moves through the playlist using the next and previous track functions, the name of the track is spoken aloud. In addition, the gesture based player provided a non-speech sound in the case of a gesture being performed that could not be recognised. Our assumption was that implicit feedback would be provided by the output of the player. The participant noted that whilst this was largely the case if a track was being played, and there were obvious increases in volume etc., no audio feedback was provided if the track was paused and the volume changed. Additionally, if the track was playing, he found it irritating that the name of the song was spoken when selecting the next track. These issues were not identified in the blindfolded study, but in that study the tasks and their sequence was more constrained than the informal interaction that occurred here. It is important therefore that audio feedback always be provided in response to user actions, but care should be taken to determine if explicit audio feedback is necessary, or if implicit feedback will suffice.

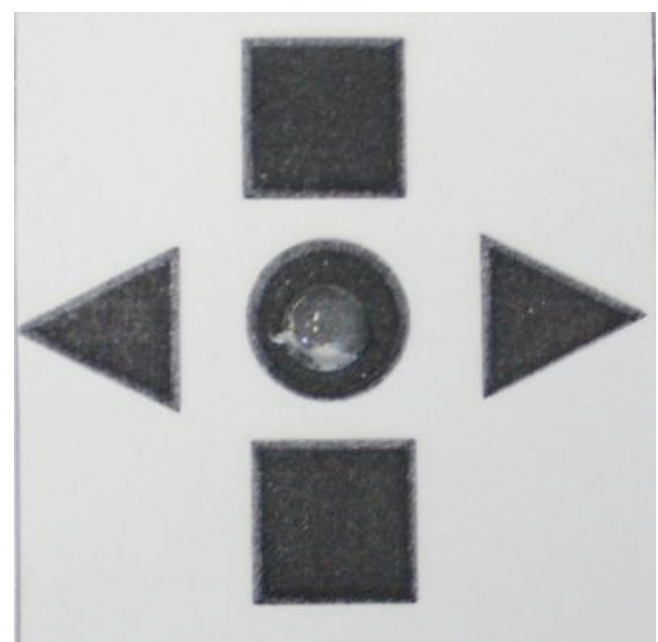

Figure 10: The control panel player with a "bump on" attached to improve location awareness.

\section{GUIDELINES FOR TOUCH SCREEN ACCESSIBILITY}

From the results of both of the evaluations, we can begin to derive guidelines for designers to employ in the future development of touchscreen accessible interfaces.

Do not use short impact related gestures (e.g. tap): Many of the problems users encountered related to simple tap gestures, or performing a tap gesture when another had been intended. It is difficult for a user to know how far above the touchscreen his or her finger is, making it easy to accidentally perform impact related gestures, or unexpectedly impact the screen causing another gesture to be performed. This occurred for example, when trying to execute play/pause whilst actually sliding and executing a volume change.

Avoid "localised" gestures or provide touchscreen awareness: A particular problem identified by the visually impaired participant, was awareness of his location on the touchscreen with respect to its borders. Therefore designers should avoid gestures that require users to start interacting on specific spatial locations of the screen. Our gestures though also suffering from this issue, only required short directional stokes, so awareness of user 
position on screen was not so much of a problem. A way around this may be to employ technology to provide awareness of the user location. Crossan and Brewster [15] used dynamic audio to make participants aware of their location in the drawing frame, and this may be possible here.

Provide a discernable tactile "home": Ensure that on any tactile control pad there is one key that can easily and quickly be identified, to allow the user to orientate his or herself within the interface. Stuck on bumps can be used to achieve this.

Use different button shapes: In addition to a tactile home key, using different shapes of button is effective to allow users to quickly, and easily, identify location and functionality. Also ensure that buttons are of adequate size, and are sufficiently separated so that they are not confused. A sizing of $1.5 \mathrm{~cm}$ on each side, and $0.5 \mathrm{~cm}$ minimum separation is a good guide.

Provide feedback for all actions: All interactions should have some sort of feedback. From discussions with the visually impaired participant, audio is the preferred option. Implicit feedback (e.g. increasing the volume in response to a volume change command) can be used instead of explicit audio feedback (such as reading the next track name in response to a next track command). In our study, implicit feedback, if available, was preferred, as the participant did not want the names of the tracks read out when switching between them, as playing of the next track would have sufficed. Care should be taken over situations where no implicit feedback is generated. In such cases explicit feedback should be used.

\section{FUTURE WORK}

Whilst the gesture interface offers promise, further work is needed to identify the kinds of gestures that are suitable, and to provide some dynamic feedback to assist the user. The buttons, although more popular and consistent with current interfaces, do have problems. The need to carry around one or more overlays would be an issue. Our future work is focused on providing the same functionality of the buttons interface, but without the need of a separate overlay. Instead of using a paper based overlay to provide a tactile relief, we will investigate using a piezoelectric screen. A piezoelectric screen employs a small vibrotactile actuator under the touchscreen which can be triggered to vibrate and provide useful feedback to the user on button selection [20]. These screen are becoming more common, though have been little used up to now, and provide a lower latency between activation and when the vibration is felt by the user, than existing vibration motors. We intend to investigate if this technology can provide a virtual control panel that can be felt and discerned without sight. However, existing touchscreens have only two states, active and inactive. This caused problems in our system with accidental touches triggering gestures. In order to feel controls without accidental activation, another technique would be needed. Our intention is to use pressure based feedback, which rather than having an active/inactive binary state, provides information on how hard the user is pressing the screen. This technology is common in graphics tablets, but less so on other devices. The only mobile device that currently supports pressure based input is the Nokia N800 internet tablet (see Figure 11). Currently there are no devices that support both piezoelectric screens and pressure based input. However as such devices become more available, new possibilities for touchscreen interaction will become possible.

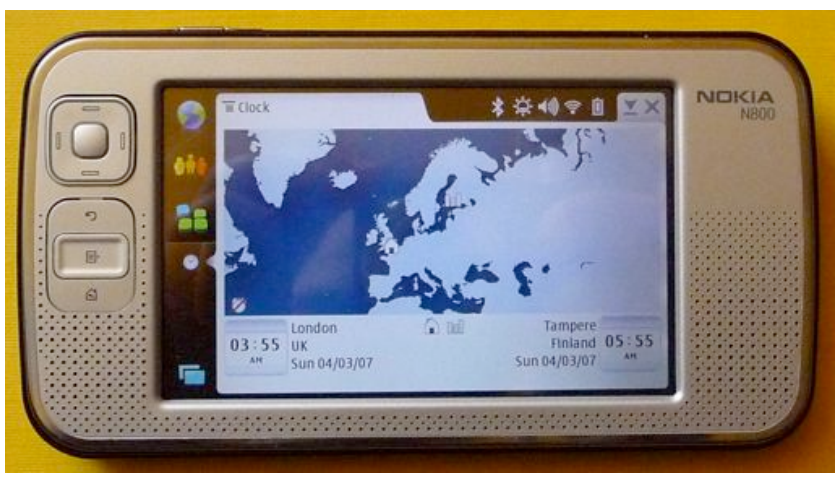

Figure 11: The Nokia N800 Internet Tablet incorporates a pressure sensitive display.

\section{CONCLUSIONS}

Through evaluation of both the gesture and control panel based touchscreen MP3 players, we have explored how touchscreen based technology can be made more accessible for those with visual impairments. Both approaches have shown promise and issues. The gestures, whilst being slower and less accurate, as well as raising questions over how much the gesture set could be increased, were usable by participants. Many of the problems emanated from the tapping gesture used to pause and play tracks. The gesture technique is also the "cleanest" solution. Using control panels, such as the buttons interface, requires some form of overlay to be placed between the surface of the touchscreen and the user's finger. Whilst for the PDA that we used this did not present problems, as position is detected through physical force being applied on the screen, other technologies to detect touchscreen position (such as a capacitance screen as used on the iPhone) may not work correctly if there is an overlay between the screen and the user. The approach suggested by the visually impaired user, of "inverting" the control panel where buttons are recessed instead of raised, may help here, but further investigation is needed to determine if such a control panel would be useful. Additionally with the buttons, the user would need to carry around a tactile overlay. We have already discussed how the system may detect the presence of the overlay automatically, but it would still be something that would need to be supplied and carried around. If each interface required a different control panel, this would likely make the overlay technique impractical.

We can conclude that in all cases the visual touchscreen interface would need to be adapted to accommodate the needs of a visually impaired person. However, we have achieved our aim of avoiding permanent physical modification to the device. This means that it would be possible to build a single device that could be adapted to serve both visually impaired and sighted users, thus reducing the separation when choosing between the appropriated and assistive technology identified from the questionnaire results. Both of the techniques investigated here are promising, but further work is required to refine their application to the scenarios discussed. However, from this work we can begin to look towards building bridges between touchscreen interaction technologies, and their use by visually impaired users. 


\section{ACKNOWLEDGMENTS}

This work was supported by EPSRC grant GR/S86150/01, EU fp6 OpenInterfaces (IST-35182) and EU fp7 HaptiMap (ICT-224675).

\section{REFERENCES}

[1] Tech Fresh. Nokia achieve - the dual-touchscreen concept phone. 2008 [cited 2008 18th April]; Available from: http://www.techfresh.net/gadgets/cell-phones/nokia-achievethe-dual-touchscreen-concept-phone/.

[2] Microsoft Corporation. Microsoft surface. 2008 [cited 2008 18th April]; Available from: http://www.microsoft.com/surface/index.html.

[3] World Health Organisation. Magnitude and causes of visual impairment. 2008 [cited 2008 19th April]; Available from: http://www.who.int/mediacentre/factsheets/fs282/en/.

[4] U.S. Department of Justice. Ada home page - ada. Gov information and technical assistance on the Americans with disabilities act. 2008 [cited 2008 18th April]; Available from: http://www.ada.gov/.

[5] Dawe, M. Desperately seeking simplicity: How young adults with cognitive disabilities and their families adopt assistive technologies. in CHI 2006. 2006. Montreal, Canada: ACM Press, 1143-1152.

[6] Morley, S., Digital talking books on a PC: A usability evaluation of the prototype daisy playback software, in Proceedings of the third international ACM conference on Assistive technologies. 1998, ACM: Marina del Rey, California, United States.

[7] Shinano Kenshi Co. Ltd. Plextalk home page. 2008 [cited 2008 18th April]; Available from: http://www.plextalk.com/in/index.html.

[8] McGookin, D. and Brewster, S. Graph builder: Constructing non-visual visualisations. in BCS-HCI 2006. 2006. London, UK: Springer,

[9] Challis, B.P. and Edwards, A.D.N., Design principles for tactile interaction. Haptic HCI 2000, 2001. 2058: p. 17-24.

[10] Wall, S. and Brewster, S.A. Tac-tiles: Multimodal pie charts for visually impaired users. in NordiChi 2006. 2006. Oslo, Norway: ACM Press,9-18.
[11] RNIB. Talking tactile tablet - $t 3$ from royal national college for the blind. 2008 [cited 2008 18th April]; Available from: http://www.rncb.ac.uk/t3/index.html.

[12] Pirhonen, A., Brewster, S., and Holguin, C. Gestural and audio metaphors as a means of control or mobile devices. in CHI 2002. 2002. Minneapolis, USA: ACM Press,291-298.

[13] Klok, M., et al. Blind gestural interaction: An experimental approach. in The 6th international workshop on Gesture in Human-Computer Interaction and Simulation. 2005. Berder Island, France18-20.

[14] Sensable Inc. Phantom omni. 2008 [cited 2008 18th April]; Available from: http://www.sensable.com/haptic-phantomomni.htm.

[15] Crossan, A. and Brewster, S., Multimodal trajectory playback for teaching shape information and trajectories to visually impaired computer users. to appear in ACM Transactions on Accessible Computing, 2008.

[16] Wobbrock, J.O. and Myers, B.A. Trackball text entry for people with motor impairments. in CHI 2006. 2006. Montreal, Canada: ACM Press,479-488.

[17] Leonard, V.K., Jacko, J.A., and Pizzimenti, J.J. An exploratory investigation of handheld computer interaction for older adults with visual impairments. in Assets'05. 2005. Balitimore, Maryland: ACM Press,12-19.

[18] Petrie, H., et al. Remote usability evaluations with disabled people. in CHI 2006. 2006. Montreal, Canada: ACM Press, 1133-1141.

[19] Kildal, J. and Brewster, S. Ema-tactons: Vibrotactile external memory aids in an auditory display. in IFIP Interact 2007. 2007. Rio de Janerio, Brazil: IEEE,71-84.

[20] Hoggan, E., Brewster, S., and Johnston, J. Investigating the effectiveness of tactile feedback for mobile touchscreens. in CHI 2008. 2008. Florence, Italy: ACM Press,1573-1582. 